\title{
Torque and torque components in high-speed permanent-magnet synchronous machines with a shielding cylinder
}

\author{
Bert Hannon $^{\mathrm{a}, \mathrm{b}}$, Peter Sergeant ${ }^{\mathrm{a}, \mathrm{b}}$, Luc Dupré ${ }^{\mathrm{b}}$ \\ ${ }^{a}$ Department of Industrial Technology and Construction, Electrical Energy Research \\ Group, Ghent University, V. Vaerwyckweg 1, 9000 Gent, Belgium \\ ${ }^{b}$ Department of Electrical Energy, Systems and Automation, Electrical Energy \\ Laboratory, Ghent University, Sint-Pietersnieuwstraat 41, 9000 Gent, Belgium
}

\begin{abstract}
A demand for more efficient electrical machines with a high power density is driving the interest for high-speed permanent-magnet synchronous machines (PMSMs). However, the design of such machines is a challenging task. One of the problems is that the effect of the shielding cylinder, a conductive sleeve around the magnets, on the machine's performance has not been studied extensively. To cope with that problem the authors of this work introduce an analytical method to study the torque in high-speed PMSMs. The presented method implies dividing the torque in two components, depending on how they are produced. The method is successfully validated and an illustration of its advantages is provided.
\end{abstract}

Keywords: High-speed machines, Analytical modeling, Torque production, Shielding cylinder

PACS: $84.50 .+\mathrm{d}$ Electric motors

\section{Introduction}

Two trends in modern society have sparked the interest in high-speed electrical machines. At the one hand an increasing ecological awareness has led to a demand for more efficient machines. At the other hand a trend towards more flexible applications pushes the demand for smaller machines. Since high-speed electrical machines, and more specifically high-speed permanentmagnet synchronous machines (PMSMs), combine a high efficiency with a 
great power density, the interest in such machines is on the rise. This is reflected in a large number of publications on high-speed machines. Some of these articles discuss specific aspects of the electrical machine, such as the shielding cylinder (SC) [12], while other articles present a more general view $[1,[2,10,15]$. A great number of publications presents a specific application or design [8, 7].

However, designing high-speed PMSMs is a challenging task. One of the reasons is that only little is known about the effects of the SC, which is commonly used in high-speed PMSMs. The SC is a conductive sleeve that is wrapped around the magnets. The reasons to implement a SC are twofold [13].

Firstly, the magnets in high-speed machines experience high centrifugal forces. They are therefore kept in position with a retaining sleeve. Usually this sleeve has a relative magnetic permeability that is near to one. The electrical conductivity of such a retaining sleeve varies from very high, if for example inconel is used, to very low, if carbon fiber is used.

Secondly, operating PMSMs at high temperatures implies a risk of permanent demagnetization of the magnets. This is particularly true for highspeed PMSMs where cooling of the rotor is difficult. Therefore the rotor losses should be minimized. A commonly proposed technique to do so is the SC. Asynchronous harmonics in the machine's magnetic field will induce eddy-currents in the conductive sleeve. According to Lenz's Law, these eddy-currents will produce a magnetic field that counteracts their origin. The asynchronous harmonics in the magnetic field will thus be mitigated by the SC. This means that, when designed correctly, the SC reduces the overall rotor losses [6, 13].

To get a better understanding of the effects of the shielding cylinder on the machine's performance, this work studies the torque in PMSMs that are equipped with a SC. The novelty of the work includes the separation of the torque in two components, as explained in Section 4. There are two major techniques to study the torque in electrical machines. One possibility is to build a finite-element model (FEM) of the studied machine. Such models are very accurate and relatively easy to construct. However, if the goal is to get a better understanding of the machine's physics, analytical models are better suited. Not only because of their low computational times and great flexibility but also because they intrinsically lend themselves to getting a better understanding of the impact of the chosen parameters. Therefore in this work an analytical model is used to study the torque. 
To conclude this introduction an outline of the presented work is given in the following.

In Section 2 the studied machine topology is presented. Section 3 introduces the analytical model that was applied in the torque study. It also presents the assumptions that where adopted to enable the analytical approach. In Section 4 the torque production in high-speed PMSMs is discussed and the division of the torque in two components is presented. That section also discusses the calculation of the torque based on the model presented in Section 3. Before concluding the work in Section 6. Section 5 demonstrates the advantages of the proposed division in torque components.

\section{Machine geometry}

The machine type regarded in this study is a PMSM that is equipped with a SC. The cross section of such a machine, with 3 phases $(m=3)$, 2 pole pairs $(p=2)$ and 12 slots $\left(N_{s}=12\right)$, is shown in Figure 1. The machine's geometrical parameters are $r_{1}$ (outer radius of the rotor yoke), $r_{2}$ (outer radius of the magnets), $r_{3}$ (outer radius of the $\mathrm{SC}$ ), $r_{4}$ (inner radius of the stator windings), $r_{5}$ (outer radius of the stator windings) and $r_{6}$ (outer radius of the machine). The magnet span is $\varphi_{m}$ and the slot opening is $\delta$. The angular position of the $i^{\text {th }}$ slot is:

$$
\delta_{i}=-\frac{\delta}{2}+\frac{2 \pi}{N_{s}} i \quad \text { with } 1 \leq i \leq N_{s}
$$

where $N_{s}$ is the number of slots.

The shaded areas in Figure 1 depict the teeth. In a slotted machine these teeth are made of a highly permeable material such as electrical steel. In a slotless machine the teeth consist of a material with a relative permeability that approximates 1 . Notice that the modeled machine does not contain any stator tips. While taking into account the stator tips would increase the computational time, it would not significantly increase the accuracy since saturation is neglected in this work. Depending on the goal of the performed study, the slot opening in the model can be adjusted to match the slot opening or the stator-tip opening of the studied machine.

\section{2D analytical subdomain model}

The literature describes a large number of modeling techniques for various machine types, extensive reviews of the existing literature can be found in 


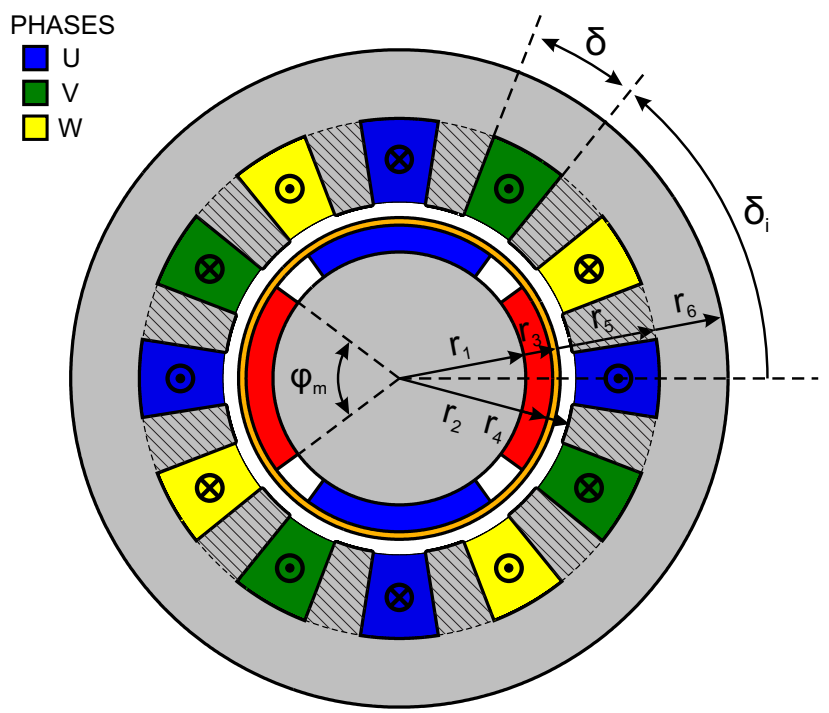

Figure 1: Geometry of a 3-phase PMSM with 2 pole pairs and a SC

[14, 3]. The model adopted here is a $2 \mathrm{D}$ analytical subdomain model. It calculates the magnetic vector potential (A), which is defined as:

$$
\mathbf{B}=\nabla \times \mathbf{A}
$$

The model accounts for the slots and the eddy-current reaction field. As shown in Section 4, the torque may then be calculated from the magnetic vector potential by applying Maxwell's stress tensor.

The model has been developed by the authors and is extensively discussed and validated in [5]. It will be introduced in the following. However, first the adopted assumptions are listed.

\subsection{Assumptions}

The following assumptions are made to enable an analytical approach:

- infinite permeability of the rotor and stator iron

- relative permeability of the $\mathrm{SC}$ and stator slots $\mu_{r}=1$

- zero conductivity of the magnets and the rotor and stator iron

- no induced currents in the slots 
- radial slot boundaries

- no end effects

Neglecting the conductivity in the magnets and the rotor and stator iron implies applying the resistance-limited approximation [6, 11].

\subsection{D analytical subdomain model}

The subdomain technique divides the studied geometry in a number of regions, called subdomains. The idea is that the problem is simplified when regarded separately in each of these regions. The solutions, obtained in the individual subdomains, are then linked back together by imposing physical boundary conditions. This approach has the advantage of great accuracy and a good insight in the machine's physics. The latter is especially true because the results are available separately for every combination of the time and spatial harmonics, as shown in (17) and (8).

In this paper the subdomains are chosen as illustrated in Fig. 2, They are indicated with an index $\nu$. The magnet subdomain is represented by $\nu=1$, 2 stands for the SC and 3 for the air gap. In slotless machines the entire winding region is regarded as one subdomain and is indicated with an index 4. In slotted machines every slot is a separate subdomain, indicated with an index $4 i$. Where $i$ is the slot number.

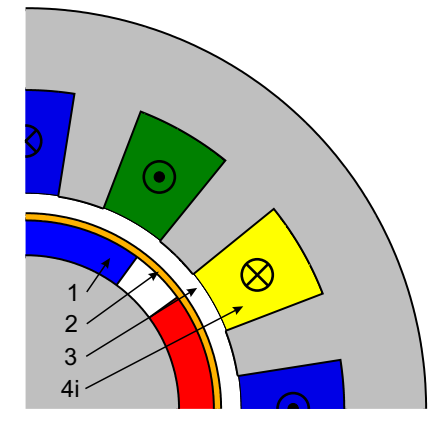

(a) Slotted topology

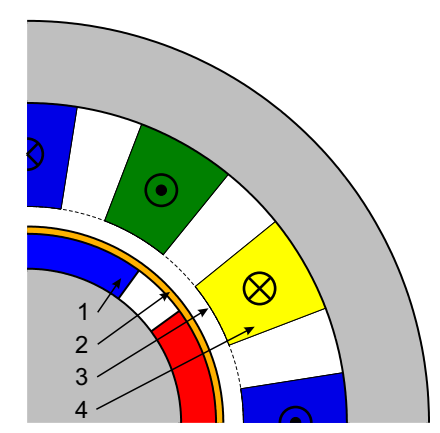

(b) Slotless topology

Figure 2: Subdomains in the studied topologies

\subsection{Govering equation}

Based on Maxwell's equations and the constitutive relations a differential equation for the magnetic vector potential can be proposed [6]. In subdomain 
$\nu$ this equation can be written as:

$$
-\Delta \mathbf{A}^{(\nu)}+\mu_{\nu} \sigma_{\nu} \frac{\partial \mathbf{A}^{(\nu)}}{\partial t}=\mu_{\nu} \mathbf{J}_{\text {ext }}^{(\nu)}+\nabla \times \mathbf{B}_{\text {rem }}^{(\nu)}
$$

Where $\mathbf{J}_{\text {ext }}^{(\nu)}$ is an externally imposed current density, $\mathbf{B}_{\text {rem }}^{(\nu)}$ stands for the remanent magnetic induction, $\mu_{\nu}$ is the permeability and $\sigma_{\nu}$ the conductivity. This equation is indeed greatly simplified in the different subdomains, the source terms $\mathbf{B}_{\text {rem }}^{(\boldsymbol{\nu})}$ and $\mathbf{J}_{\text {ext }}^{(\boldsymbol{\nu})}$ only appear in the magnet and winding regions respectively. The time-derivative term accounts for induced currents, these currents are only considered in the SC.

The boundary conditions, imposed on every boundary between two subdomains, are conservation of the magnetic flux and Ampère's law. These conditions can be written in terms of the vector potential as:

$$
\mathbf{A}^{(\nu)}=\mathbf{A}^{(\nu+1)}
$$

and

$$
\mathbf{n} \times\left(\mathbf{H}^{(\nu)}-\mathbf{H}^{(\nu+1)}\right)=\mathbf{K}^{(\nu)}
$$

Where $\mathbf{n}$ is the unit vector along the normal direction and $\mathbf{K}^{(\boldsymbol{\nu})}$ the current density on the boundary.

In the following a cylindrical coordinate system, $(r, \varphi, z)$, fixed to the rotor and with the z-axis along the machine's axis, is used. By neglecting the end effects, the problem can be regarded in a plane perpendicular to the $z$-axis. The magnetic vector potential is then independent of $z$ and has no $r$ - or $\varphi$-component. Mathematically the magnetic vector potential can now be written as:

$$
\mathbf{A}^{(\nu)}=A^{(\nu)}(r, \varphi, t) \cdot \mathbf{e}_{\boldsymbol{z}}
$$

\subsection{Vector potential equation}

The above results in equations for the magnetic vector potential in each of the subdomains. As explained in [5], the equations for the magnetic vector potential in the slots have a different spatial periodicity than the ones in the other subdomains. The magnetic vector potential in a slotless machine can be written as:

$$
A^{(\nu)}(r, \varphi, t)=\sum_{n=-\infty}^{\infty} \sum_{k=-\infty}^{\infty} A_{n, k}^{(\nu)}(r) e^{j\left(k \varphi+(k-n) \omega_{m} t+k \varphi_{0}\right)}
$$


Where $\omega_{m}$ is the mechanical pulsation, $\varphi_{0}$ is the initial angle of the rotor with respect to the stator, $n$ represents the time harmonic order and $k$ is the spatial harmonic order. The form of $A_{n, k}^{(\nu)}(r)$ depends on the differential equation in the $\nu^{\text {th }}$ subdomain. In slotted machines the magnetic vector potential is written as:

$$
\begin{aligned}
& A^{(\nu)}(r, \varphi, t)= \\
& \begin{cases}\sum_{n=-\infty}^{\infty} \sum_{k=-\infty}^{\infty} A_{n, k}^{(\nu)}(r) e^{j\left(k \varphi+(k-n) \omega_{m} t+k \varphi_{0}\right)} & \text { if } \nu=1 . .3 \\
\sum_{n=-\infty}^{\infty} \sum_{l=-\infty}^{\infty} A_{n, l}^{(\nu)}(r) e^{j\left(\frac{l \pi}{\delta}\left(\varphi-\delta_{i}\right)+\left(\frac{l \pi}{\delta}-n\right) \omega_{m} t+\frac{l \pi}{\delta} \varphi_{0}\right)} & \text { if } \nu=4 i\end{cases}
\end{aligned}
$$

To avoid confusion the spatial harmonic order in the slots was given a different denotation than the spatial harmonic order in the other regions, $l$ instead of $k$. Note that the vector potential is available for every time and spatial harmonic combination, $(n, k)$ or $(n, l)$, separately.

\section{Torque calculation}

In a machine that is equipped with a shielding cylinder torque is produced in two ways.

A first component of the torque is produced due to the interaction between the magnets and the stator currents. It is referred to as the torque produced in the magnets $\left(T_{P M}\right)$. This component of the torque is dominated by the synchronous harmonic combinations. These are harmonic combinations that are independent of time when referred to the rotor. From (77) and (8) it can be seen that this implies an equal time and spatial harmonic order $(n=k)$. Indeed, the magnets themselves are time-invariant with respect to the rotor and will thus only introduce synchronous harmonic combinations.

A second torque component is the result of interaction between the stator currents and the eddy currents in the shielding cylinder, it is referred to as the torque produced in the $\mathrm{SC}\left(T_{S C}\right)$. This torque will be dominated by asynchronous harmonic combinations. Indeed, synchronous combinations do not induce eddy-currents in the SC. Note that the SC can be regarded as the squirrel cage of an induction machine. The slip with respect to the synchronous combinations is then zero, only asynchronous harmonic combinations can produce torque. 
$T_{P M}$ and $T_{S C}$ are fictitious torque components, they do not occur separately. Nevertheless, regarding these components separately can be very useful to get a better understanding of the machine's physics, as shown in Section 5 .

\subsection{Torque calculation}

The torque acting on a certain volume can be computed by integrating the cross product of the radius and Maxwell's stress tensor $(\boldsymbol{\Gamma})$ over the surface that volume:

$$
\mathbf{T}=\oint_{S} \mathbf{r} \times \mathbf{\Gamma} \mathrm{d} s
$$

where the stress tensor is defined as:

$$
\boldsymbol{\Gamma}=\mu_{0}(\mathbf{n} \cdot \mathbf{H}) \mathbf{H}-\frac{\mu_{0}}{2}(\mathbf{H} \cdot \mathbf{H}) \mathbf{n}
$$

$\mathbf{H}$ is the magnetic field strength and $\mathbf{n}$ is the outer unit vector normal to the integration surface $S$. The radial component of the torque acting on the entire rotor $(T)$ can then be calculated by choosing the integration surface as a cylinder with radius $r_{3}$ and an axis that is coaxial to the machine's axis, see Figure 3a,

$$
\begin{aligned}
T(t) & =\frac{l_{s} r_{3}^{2}}{\mu_{0}} \int_{0}^{2 \pi} B_{r}^{(3)}\left(r_{3}, \varphi, t\right) B_{\varphi}^{(3)}\left(r_{3}, \varphi, t\right) \mathrm{d} \varphi \\
& =2 \pi \frac{l_{s} r_{3}^{2}}{\mu_{0}} \sum_{n=-\infty}^{\infty} \sum_{s=-\infty}^{\infty} T_{n, s} e^{-j(n+s) \omega_{m} t}
\end{aligned}
$$

Where $n$ is the time harmonic order related to $B_{r}^{(3)}, s$ is the time harmonic order related to $B_{\varphi}^{(3)}$ and $T_{n, s}$ is calculated as:

$$
T_{n, s}=\sum_{k=-\infty}^{\infty} B_{r, n, k}^{(3)}\left(r_{3}\right) B_{\varphi, s,-k}^{(3)}\left(r_{3}\right)
$$

The radial and tangential components of the flux density are calculated by applying (2):

$$
\begin{aligned}
B_{r, n, k}^{(3)} & =\frac{1}{r} \frac{\partial A_{n, k}^{(3)}(r, \varphi, t)}{\partial \varphi} \\
B_{\varphi, s, k}^{(3)} & =-\frac{\partial A_{s, k}^{(3)}(r, \varphi, t)}{\partial r}
\end{aligned}
$$


The torque produced in the magnets can be calculated in a similar way. However, now a cylinder with radius $r_{2}$ is chosen as the integration surface. This is illustrated in Figure 3b, $T_{P M}$ can then be calculated as:

$$
T_{P M}(t)=2 \pi \frac{l_{s} r_{2}^{2}}{\mu_{0}} \sum_{n=-\infty}^{\infty} \sum_{s=-\infty}^{\infty} T_{P M, n, s} e^{-j(n+s) \omega_{m} t}
$$

Where $T_{P M, n, s}$ is calculated as:

$$
T_{P M, n, s}=\sum_{k=-\infty}^{\infty} B_{r, n, k}^{(1)}\left(r_{2}\right) B_{\varphi, s,-k}^{(1)}\left(r_{2}\right)
$$

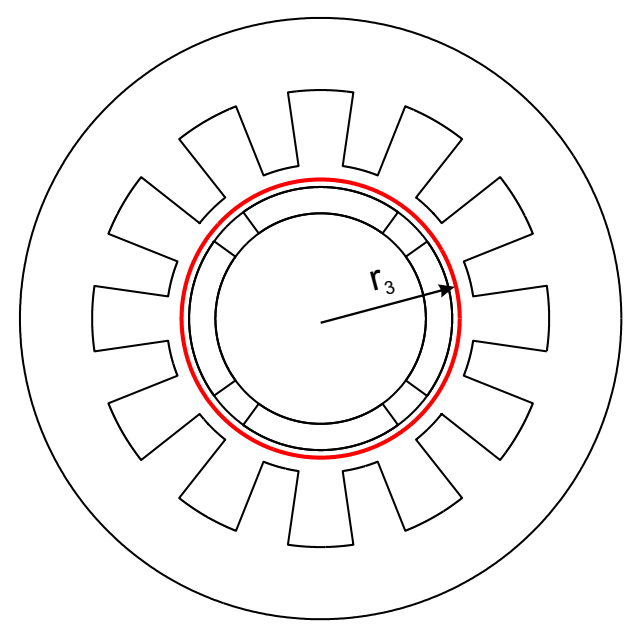

(a) Integration surface for $T$

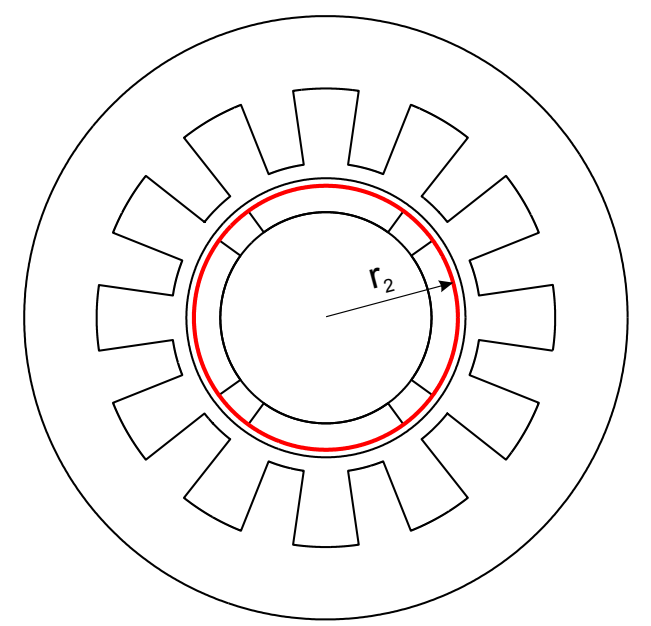

(b) Integration surface for $T_{P M}$

Figure 3: Integration surface for torque calculation

Since $T_{P M}$ and $T_{S C}$ are the only torque components that make up $T$, the torque produced in the shielding cylinder can be calculated as in (16).

$$
T_{S C}=T-T_{P M}
$$

\subsection{Validation}

In order to validate the equations presented in this section a validation using a FEM is presented. The test was performed for a slotted machine similar to the one depicted in Figure 1. The machine's parameters are listed 
in Table 1. The highest order of the time and spatial harmonics has been limited to 80 , the radially magnetized and the applied current is a balanced sinusoidal system with 3 phases.

The resulting torque components, $T, T_{P M}$ and $T_{S C}$, are shown in Figure

Table 1: Parameters of the studied machine

\begin{tabular}{lll}
\hline Symbol & Parameter & Value \\
\hline$r_{1}$ & Rotor yoke radius & $14.0 \mathrm{~mm}$ \\
$r_{2}$ & Permanent magnet radius & $17.5 \mathrm{~mm}$ \\
$r_{3}$ & Shielding cylinder radius & $18.5 \mathrm{~mm}$ \\
$r_{4}$ & Air gap outer radius & $20.5 \mathrm{~mm}$ \\
$r_{5}$ & Slot outer radius & $30.4 \mathrm{~mm}$ \\
$r_{6}$ & Machine outer radius & $40.0 \mathrm{~mm}$ \\
$l_{s}$ & Stack length & $200.0 \mathrm{~mm}$ \\
$N$ & Number of slots & 12 \\
$w$ & Number of windings per slot & 5 \\
$\delta$ & Slot opening angle & $0.55 \frac{2 \pi}{N} \mathrm{rad}$ \\
$B_{r e m}$ & Remanent magnetic induction & $1.2 \mathrm{~T}$ \\
$p$ & Number of pole pairs & 2 \\
$\varphi_{m}$ & Magnet span & $0.8 \frac{\pi}{p} \mathrm{rad}$ \\
$I$ & Externally imposed current (amplitude) & $72,5 \mathrm{~A}$ \\
$f$ & Electrical frequency & $1000 \mathrm{~Hz}$ \\
$\sigma_{2}$ & Conductivity of the SC & $5.96 \cdot 10^{7} \Omega \mathrm{m}$ \\
\hline
\end{tabular}

4. There is a very good agreement between the results obtained from the analytical model and the FEM. Note that there is a slight offset between the $T_{P M}$ obtained from FEM and the $T_{P M}$ obtained from the analytical model. It is assumed that this is due to the fact that, in contrast to the analytical model, the FE model accounts for a finite permeability of the iron.

\section{Parameter study}

To illustrate the benefit of distinguishing two torque components, this section presents a parameter study of $T, T_{P M}$ and $T_{S C}$. The machine that is being studied is the same machine that was used for the validation in Section 4.2. All of the results presented in the following are obtained with the help 

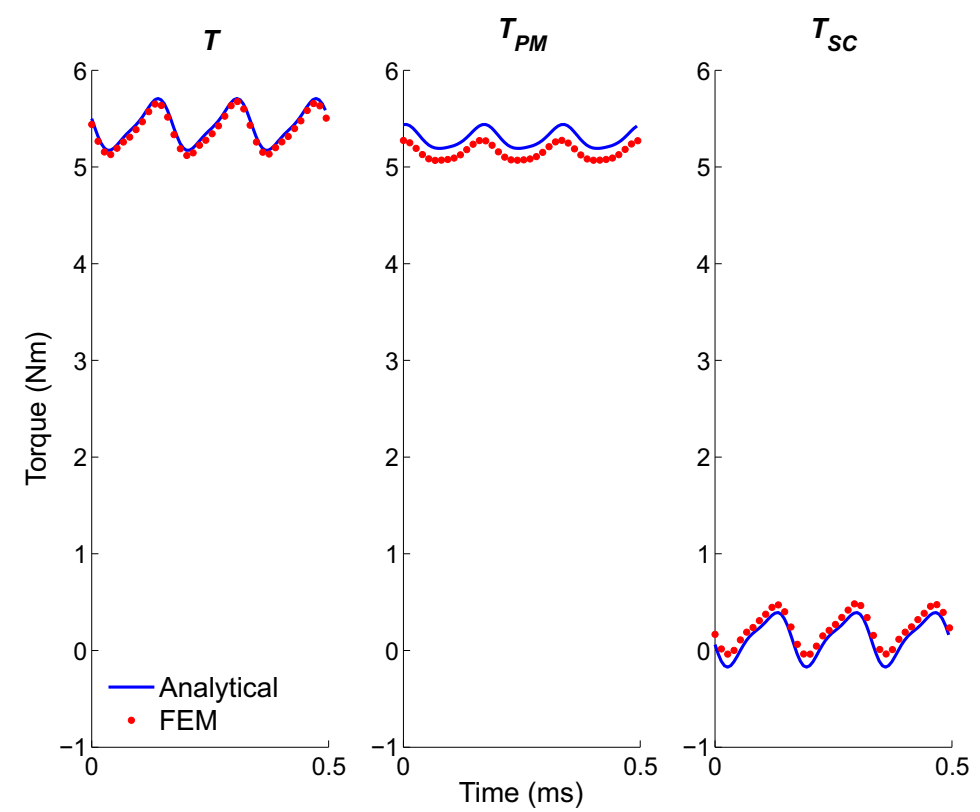

Figure 4: Validation of the calculated torque components

of the analytical model. The parameter that is varied here is the opening angle of the slot $(\delta)$. To do so the slot-opening coefficient $(\alpha)$ is defined as the ratio between the opening angle of the slots and the tooth pitch:

$$
\alpha=\frac{\delta}{\frac{2 \pi}{N_{s}}}
$$

Two aspects of the torque will be regarded, the net torque level and the torque ripple. The net torque levels are indicated with a subscript net; $T_{n e t}$, $T_{P M, n e t}$ and $T_{S C, n e t}$ respectively. The torque ripple, indicated with a prefix $\Delta$ and a subscript $\%$, is expressed as a percentage of $T_{\text {net }}$ :

$$
\left\{\begin{array}{l}
\Delta T_{\%}=\frac{\max (T)-\min (T)}{T_{n e t}} \cdot 100 \% \\
\Delta T_{P M, \%}=\frac{\max \left(T_{P M}\right)-\min \left(T_{P M}\right)}{T_{n e t}} \cdot 100 \% \\
\Delta T_{S C, \%}=\frac{\max \left(T_{S C}\right)-\min \left(T_{S C}\right)}{T_{n e t}} \cdot 100 \%
\end{array}\right.
$$

A similar study has been performed by Güemes et al. in [4]. However, their study considered a conventional PMSM and was performed using a FEM. 
Since the studied machine did not contain a SC, no division of the torque was considered. Note that the goal here is to perform a theoretical study, some of the proposed machines would not be built in practice.

\subsection{Net torque}

While keeping all the other parameters constant, $\alpha$ is increased from 0.35 to 0.95 in steps of 0.10 .

An increasing $\alpha$ results in a higher Carter factor $\left(k_{c}\right)[9]$ :

$$
k_{c}=\frac{1}{1-\sigma_{c} \alpha}
$$

with:

$$
\sigma_{c}=\frac{2}{\pi}\left(\arctan \frac{d_{s}}{2 l_{a}}-\frac{2 l_{a}}{d_{s}} \ln \sqrt{1+\left(\frac{d_{s}}{2 l_{a}}\right)^{2}}\right)
$$

where $l_{a}$ is the length of the air gap $\left(l_{a}=r_{4}-r_{3}\right)$ and $d_{s}$ is the width of the slot at the border with the air gap $\left(d_{s}=\delta r_{4}\right)$.

The expectation is that the torque will decrease in accordance with the increasing Carter factor. The dashed blue line in Figure 5 shows the torque prediction using Carter's factor. This prediction was done by regarding the torque at $\alpha=0.65$ as a reference point. It can be seen that, despite the expectation, the difference between the predicted torque and the actual torque is unacceptably high. However, if the same technique is used to predict $T_{P M}$ the accuracy is much higher. It seems that the torque produced in the magnets indeed suffers from a higher carter factor.

In contrast to $T_{P M}$, the torque produced in the shielding cylinder is mainly dependent on the asynchronous components of the magnetic field. The amplitude of these components depends, amongst others, on the slotting effect, which in turn depends on $\alpha$ and the depth of the slots. It can thus be concluded that $T_{S C}$ strongly depends on the slotting effect.

Initially, the expected decrease of $T_{P M}$ due to a higher Carter factor is compensated due to a larger slotting effect, and thus a higher $T_{S C}$. This is illustrated by the vertical green lines in Figure 5. When $\alpha$ is increased even further, the slotting effect decreases again while $k_{c}$ keeps increasing. This results in a steeper decrease of $T$. 


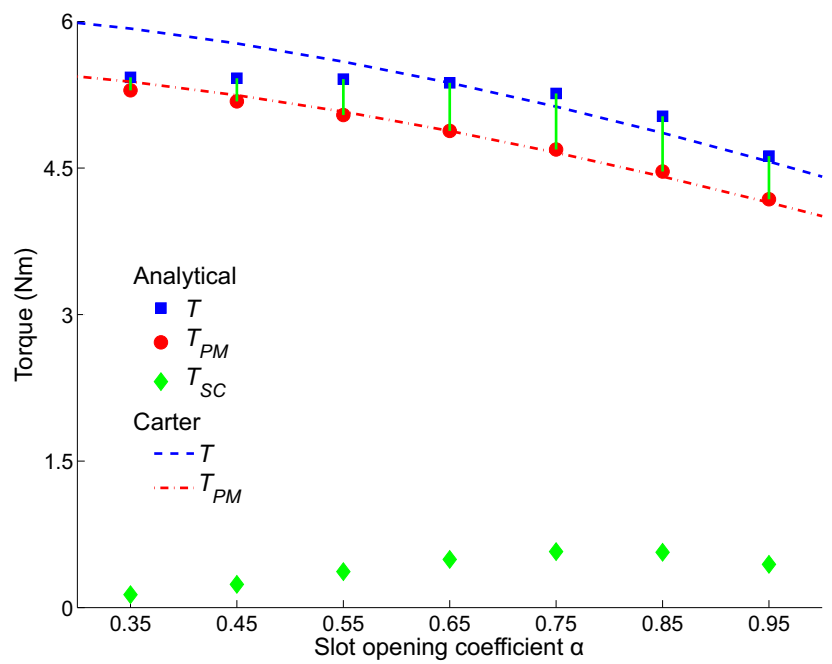

Figure 5: Evolution of the net torque levels with respect to $\alpha$

\subsection{Torque ripple}

The torque ripple is also studied by varying $\alpha$ from 0.35 to 0.95 in steps of 0.10 . The other parameters are kept constant. The expectation is that the torque ripple will be dominated by the slotting effect. This is indeed confirmed by Figure 6.

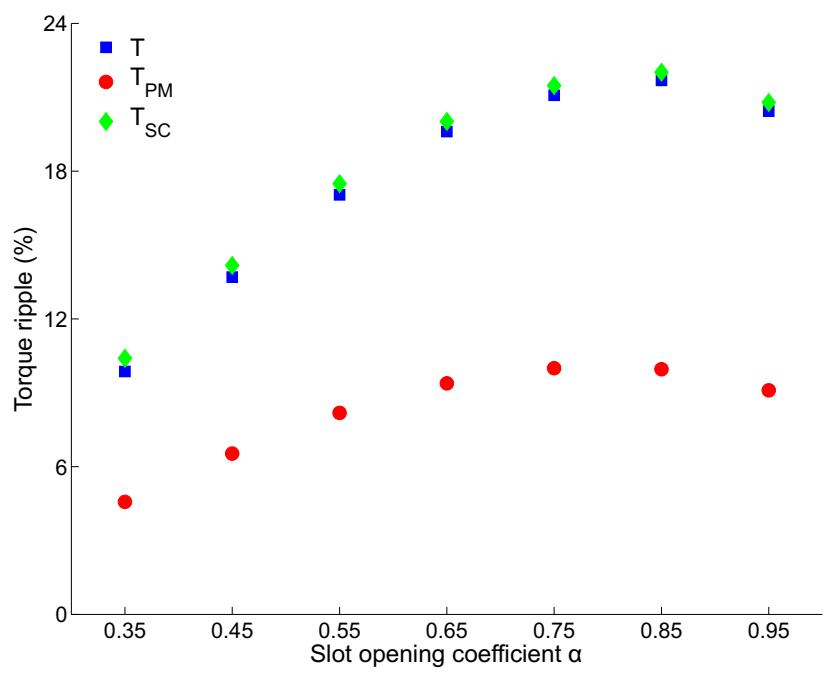

Figure 6: Evolution of the percentage of the torque ripple with respect to $\alpha$

An interesting aspect of Figure [6 is that the sum of $\Delta T_{P M, \%}$ and $\Delta T_{S C, \%}$ 
does not equal $\Delta T_{\%}$. This indicates that there is a time shift between the torque ripple produced in the magnets and the torque ripple produced in the shielding cylinder, as confirmed by Figure 7.

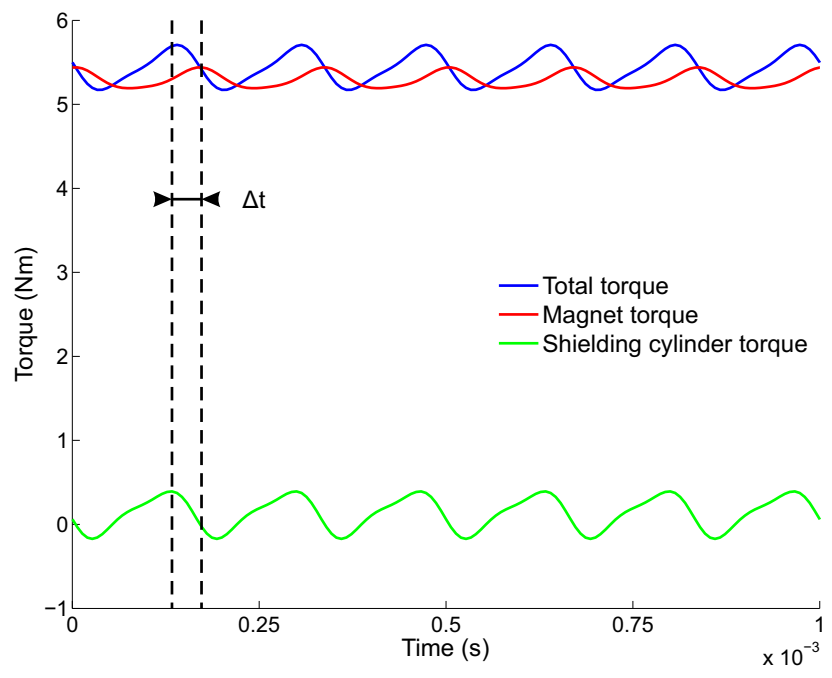

Figure 7: $T, T_{P M}$ and $T_{S C}$ in function of time

The time shift shown in Figure 7 can be explained by the different nature of $T_{P M}$ and $T_{S C}$. While $T_{P M}$ reaches a maximum depending on the alignment of the PMs with the stator teeth, $T_{S C}$ will reach a maximum depending on the rate at which the magnetic field changes. These phenomena do not necessarily happen simultaneously.

Note that this parameter study clearly demonstrates the advantages of regarding $T_{P M}$ and $T_{S C}$ separately. In Section 5.1 the evolution of the net torque was easily explained by studying both torque components separately. In Section 5.2 studying the torque ripples separately resulted in a better insight in the machine's physics. It was proven that there is a time shift between $T_{P M}$ and $T_{S C}$. This information may, for example, be used in an optimization study to reduce the torque ripple.

\section{Conclusion}

In this work an analytical method to study the torque in high-speed PMSMs has been presented. The torque has been studied using a 2D analytical subdomain model. The torque production was discussed in Section 4 and a division of the torque in two components, the torque produced in the 
magnets and the torque produced in the SC, has been proposed. A method to calculate these components was presented and validated. Finally a parameter study has been carried out to illustrate the advantages of considering two torque components.

By introducing an analytical method to study the torque components of high-speed PMSMs, this work provides a tool to get a better understanding of such machines. Moreover, because of its analytical character the tool is very fast and very flexible. It is thus also suited for optimization problems. Note that the analytical model does not account for 3D effects such as the end-effects. For that reason, it should not be used for detailed design of electric machines.

Examples of interesting studies for the future are: the effect of different materials and dimensions of the SC, optimization of the machine's geometry to reduce torque ripple, accounting for the effect of the magnet's conductivity and studying the effect of the current waveform on the machine's performance. It would also be very interesting to perform a similar study of the rotor losses.

\section{References}

[1] N. Bianchi, S. Bolognani, F. Luise, Potentials and limits of high-speed PM motors, IEEE Trans. Ind. Appl. 40 (2004) 1570-1578.

[2] A. Chebak, P. Viarogue, J. Cros, Optimal design of a high-speed slotless permanent magnet synchronous gemerator with soft magnetic composite stator yoke and rectifier load, Math. Comput. Simul. 101 (2010) 67-77.

[3] F. Dubas, A. Rahideh Two-dimensional analytical permanent-magnet eddy-current loss calculations in slotless PMSM equipped with surfaceinset magnets IEEE Trans. Magn. 50 (2014) 54-73.

[4] J.A. Güemes, P. García, A. Iraolagoitia, J. Ugartemendia, Influence of slot opening width and rotor pole radius on the torque of PMSM, Proc. Int Conf. on Renewable Energies and Power Quality 2009, Valencia Spain 2009, pp. 1-5

[5] B. Hannon, P. Sergeant, L. Dupré, 2D analytical subdomain model of a slotted PMSM with shielding cylinder, IEEE Trans. Magn. 50 (2014) $1-10$. 
[6] S.R. Holm, H. Polinder, J. Ferreira, Analytical modeling of a permanentmagnet synchronous machines in a flywheel, IEEE Trans. Magn. 43 (2007) 1955-1967.

[7] D.-K. Hong, B.-C. Woo, J.-Y. Lee, D.-H. Koo, Ultra high speed motor supported by air foil bearings for air blower cooling fuel cells, IEEE Trans. Magn. 43 (2012) 871-874.

[8] S.-M. Jang, H.-W. Cho, S.-K. Choi, Design and analysis of a high-speed brushless DC motor for centrifugal compressor, IEEE Trans. Magn. 48 (2007) 2573-575.

[9] J. Melkebeek, L. Vandevelde, Bouw en Berekening van Elektrische Machines, fifth ed., Ghent University, Ghent, 2007.

[10] P.-D. Pfiser, Y. Perriard, Very-high-speed slotless permanent-magnet motors: Analytical modeling, optimization, design and torque measurement methods, IEEE Trans. Ind. Appl. 57 (2010) 296-303.

[11] P. Sergeant, A. Van den Bossche, Influence of the amount of permanentmagnet material in fractional-slot permanent-magnet synchronous machines, IEEE Trans. Ind. Appl. 61 (2014) 4979-4989.

[12] M. Shah, S.-B. Lee, Rapid analytical optimization of eddy-current shield thickness for associated loss minimizations in electrical machines, IEEE Trans. Ind. Appl. 42 (2006) 642-649.

[13] F. Zhou, J. Shen, W. Fei, R. Lin, Study of retaining sleeve and conductive shield and their influence on rotor loss in high-speed PM BLDC motors, IEEE Trans. Magn. 42 (2006) 3398-3400.

[14] Z.Q. Zhu, L.J. Wu, Z.P. Xia An accurate subdomain model for magnetic field computation in slotted surface-mounted permanent-magnet machines IEEE Trans. Mag. 46 (2010) 1100-1115.

[15] C. Zwyssig, S. Round, J. Kolar, An ultrahigh-speed, low power electrical drive system, IEEE Trans. Ind. Electron. 55 (2008) 577-585. 